\title{
Performance comparison of different control strategies for heat exchanger networks
}

\author{
Siwaporn Mahitthimahawong1, Yada Chotvisut ${ }^{2}$, Thongchai Srinophakun,"* \\ ${ }^{1}$ Kasetsart University, Department of Chemical Engineering, 50 Ngamwongwan Rd., Ladyao, Jatujak, Bangkok 10900, \\ Thailand \\ ${ }^{2}$ King Mongkut's University of Technology Thonburi, Chemical Engineering Practice School, 126 Pracha-utid Road, \\ Bangmod, Thoongkru, Bangkok 10140, Thailand \\ "Corresponding author: e-mail: fengtcs@hotmail.com
}

\begin{abstract}
In this article, the dynamic responses of heat exchanger networks to disturbance and setpoint change were studied. Various control strategies, including: proportional integral, model predictive control, passivity approach, and passivity-based model predictive control were used to monitor all outlet temperatures. The performance of controllers was analyzed through two procedures: 1 ) inducing a $\pm 5 \%$ step disturbance in the supply temperature, or 2 ) tracking a $\pm 5^{\circ} \mathrm{C}$ target temperature. The performance criteria used to evaluate these various control modes was settling time and percentage overshoot. According to the results, the passivity-based model predictive controllers produced the best performance to reject the disturbance and the model predictive control proved to be the best controller to track the setpoint. Whereas, the ensuing performance results of both the PI and passivity controllers were discovered to be only acceptable.
\end{abstract}

Keywords: control, dynamic simulation, performance, process control, stability.

\section{INTRODUCTION}

Heat exchanger network, or more commonly known as HEN, is widely used in the chemical processing operations of industry to recover energy. Since energy conservation can significantly reduce operating costs, studying the effects of efficient recovery methods is beneficial to both small and large industry. However HEN also introduces interactions which may significantly change the overall plant dynamics, lead to slower response, increased sensitivity to disturbances and even instability. Therefore, an efficient control system is required for this operation to cause the rejection of disturbances, to follow setpoint commands effectively, and to yield a robust performance outcome.

The proportional integral (PI) control method is one of the most common approaches used to examine heat integration processes. It operates on the error signal to produce a control signal. The desired parameters are normally achieved by tuning the system to the inherent conditions without specific knowledge of a plant model ${ }^{2}$. This is not only due to its simple structure, which is conceptually easy to understand and makes manual tuning possible; but is also a method which provides adequate performance in the vast majority of applications.

Another widely accepted control technique is the powerful model predictive control (MPC) method. Its ability to handle multi-variable interactions, constraints, and optimization requirements in a systematic manner makes it a valuable asset. MPC is an online optimization-based approach that has been developed to achieve both convergence and optimal performance. Various papers deal with MPC-for example, nonlinear aspect that is characterized by the use of nonlinear system model in the prediction ${ }^{3}$, robust aspect in the presence of uncertainties ${ }^{4}$, MPC implemented on the district heating system $^{5}$. However, the MPC analysis does not guarantee closed-loop stability by itself. Several control schemes have been developed to achieve stability; many of them are based on Lyapunov stability theory ${ }^{6,7}$.
Even though the Lyapunov approach is a useful method, there is no general application for finding Lyapunov functions. As a result, trial and error are often used ${ }^{8}$. Meanwhile, the concept of passivity is simpler and has been used to design stable control systems or to show stability in control systems. To achieve a closed-loop stability and robust performance; both the passivity theorem, together with a decentralized unconditionally stable condition (DUS), must be performed ${ }^{9-12}$. In addition, a control system that combines MPC and passivity methods was developed by Raff et al. ${ }^{13}$ to take advantage of both MPC and passivity concepts.

Our objective in this work was to study the results of a dynamic simulation and to compare the closed-loop performance of PI, MPC, passivity, and passivity-based MPC control methods; in reference to disturbances and set-point changes for a heat exchanger network.

\section{MATERIAL AND METHODS}

\section{Development of heat exchanger model}

The simple heat exchanger in Figure 1 can be modeled as a series of perfect mixing under the following assumptions:

(1) Only a countercurrent heat exchanger type will be modeled because of its thermal equilibrium performance.

(2) The lump system will be considered in each mixer.

(3) There will be no phase change in order to simplify the heat transfer coefficient.

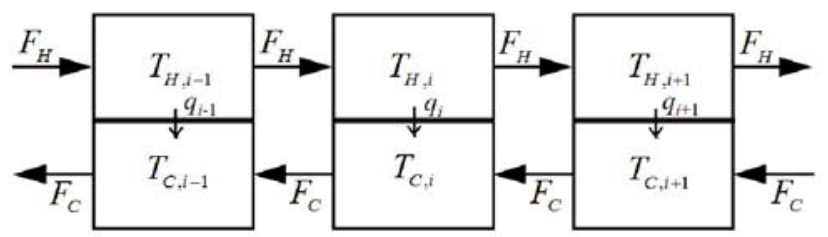

Figure 1. Heat exchanger model based on mixing tank concept 
The mathematical model of each stage (mixer) will be as

$\frac{\partial T_{H, i}}{\partial t}=\frac{F_{H}}{V_{H, i}}\left(T_{H, i-1}-T_{H, i}\right)-\frac{U A_{i}}{\rho_{H} V_{H, i} c_{V, H}} \Delta T_{m, i}$

$\frac{\partial T_{C, i}}{\partial t}=\frac{F_{C}}{V_{C, i}}\left(T_{C, i+1}-T_{C, i}\right)-\frac{U A_{i}}{\rho_{C} V_{C, i} c_{V, C}} \Delta T_{m, i}$

where $i$ represents 1 to $N$. Subscripts $H$ and $C$ are used for hot and cold streams. The variables of $T, F, V, \varrho$, and $c_{V}$ denote temperature, volumetric flow rate, volume, density, and specific heat capacity of the stream; respectively. $U$ and $A$ express the heat transfer coefficient and the exchanging area of its heat exchanger, respectively. And $\Delta T_{m}$ is the temperature driving force that is assumed to be the arithmetic mean of the temperature differences (AMTD), as shown in Eq. (3).

$\Delta T_{m, i}=0.5\left(\left(T_{H, i-1}-T_{C, i}\right)+\left(T_{H, i}-T_{C, i+1}\right)\right)$

In addition, it is required to incorporate bypass fractions into the model to control heat exchange. The outlet temperature after mixing is:

$T_{\text {out }}=f T_{\text {in }}+(1-f) T_{\text {out }}$

where $T_{\text {out }}$ is the outlet temperature prior to mixing with the bypass stream, and $f$ is the fraction of the inlet stream being bypassed from the heat exchanger on either side; hot or cold.

The state space model can be derived from the previous equations and presents itself as Eqs. (5) and (6):

$x=A x+B u+E x_{0}$

$y=C x+D u$

where $x$ is the state vector, $u$ is the manipulated vector, $x_{0}$ is the disturbance vector, $y$ is the controlled vector; and $A, B, C, D$, and $E$ are constant matrixes.

Subsequently, Equations (5) and (6) can be converted into the equivalent transfer function form. Both the process $(G(s))$ and the disturbance $\left(G_{d}(s)\right)$ transfer function matrix are presented in Eqs. (7) and (8).

$$
\begin{aligned}
& G(s)=C(s I-A)^{-1} B+D \\
& G_{d}(s)=C(s I-A)^{-1} E
\end{aligned}
$$

where $s$ is the Laplace variable and $I$ is the identity matrix.

Four control strategies were investigated, which are briefly explained here.

\section{Proportional integral control}

The manipulated variable, or control signal of a PI controller as $u(t)$ function, is calculated as follows:

$u(t)=k_{c} e(t)+\frac{k_{c}}{\tau_{I}} \int_{0}^{t} e(t) d t$

where $k_{c}$ and $\tau_{I}$ are tuning parameters and $e(t)$ is the difference between the target and outlet temperatures. The tuning parameters are calculated from the ZieglerNichols method, which is one of the most approved standards for controller tuning methods. The goal of this controller is to make $e(t)=0$, and thus eliminate the offset.

\section{Model predictive control}

The concept of MPC is to predict future behavior. At each sampling instant, an optimal control sequence is obtained from solving the following optimization problem in Eq. (10):

$$
\min _{\Delta u(k \mid k), \ldots, \Delta u(m-1+k \mid k), \varepsilon}\left(S_{y}+S_{\Delta u}+\rho_{\varepsilon} \varepsilon^{2}\right)
$$

$$
\begin{aligned}
& S_{y}=\sum_{i=0}^{p-1} \sum_{j=1}^{n_{y}}\left|w_{i+1, j}^{y}\left(y_{j}(k+i+1 \mid k)-r_{j}(k+i+1)\right)\right|^{2} \\
& S_{\Delta u}=\sum_{i=0}^{p-1} \sum_{j=1}^{n_{u}} \mid w_{i, j}^{\Delta u} \Delta u_{j}\left(\left.y_{j}(k+1 \mid k)\right|^{2}\right.
\end{aligned}
$$

s.t.

$$
\begin{aligned}
& \Delta u_{j \text { min }}(i)-\varepsilon V_{j \text { min }}^{\Delta u}(i) \leq \Delta u_{j}(k+1 \mid k) \leq \Delta u_{j \max }(i)-\varepsilon V_{j \max }^{\Delta u}(i) \\
& y_{j \text { min }}(i)-\varepsilon V_{j \text { min }}^{y}(i) \leq y_{j}(k+i+1 \mid k) \leq y_{j \max }(i)-\varepsilon V_{j \max }^{y}(i) \\
& \Delta u(k+h \mid k)=0
\end{aligned}
$$

$\varepsilon \geq 0$

where $h=m, \ldots, p-1 . p$ and $m$ are the prediction horizon and control horizon respectively. The symbol $\rho_{\varepsilon}$ is the weight on the slack variable, $\varepsilon$ represents penalizing the violation of the constraints, $r$ is the reference of the output, and $n_{y}$ and $n_{u}$ are the number of outputs and manipulated variables, respectively. Expressions $w^{y}$ and $w^{\Delta u}$ denote non-negative weights for the corresponding variable. "s.t." is an abbreviation for "subject to" means that all of the following conditions must be satisfied. $y_{\min }, y_{\max }, \Delta u_{\min }$, and $\Delta u_{\max }$ show the lower/upper bounds of the corresponding variables. $V^{y}{ }_{\min }, V^{y}{ }_{\max }$, $V_{\text {min }}^{\Delta u}$, and $V^{\Delta u}{ }_{\text {max }}$ are the Equal Concern for Relaxation (ECR) vectors that quantify the concerns for relaxing the corresponding constraints.

In Eq. (10), the predictive value is based on the information available at time $k$. The component with $(k+i \mid k)$ denotes the value predicted for time $k+i$.

Equation (11) shows that only the first element of the sequence; $\Delta u(k \mid k)$, is used to calculate the control action, $u(k)$, applied to the plant. Therefore, the remaining term of $\Delta u(k+i \mid k)$ is discarded and a new optimization problem is solved for the next sampling step of $k+1$.

$$
u(k)=u(k-1)+\Delta u(k \mid k)
$$

\section{Passivity control}

The Passivity theorem states that the closed-loop, interconnected system, comprised of a strictly passive multivariable process and a passive multi-loop controller; is decentralized unconditionally stable ${ }^{\mathbf{1 2}}$. The implementation of this concept can be summarized in Figure 2.

Foremost, how far HEN is from being passive must be determined through the calculation of a diagonal scaling 


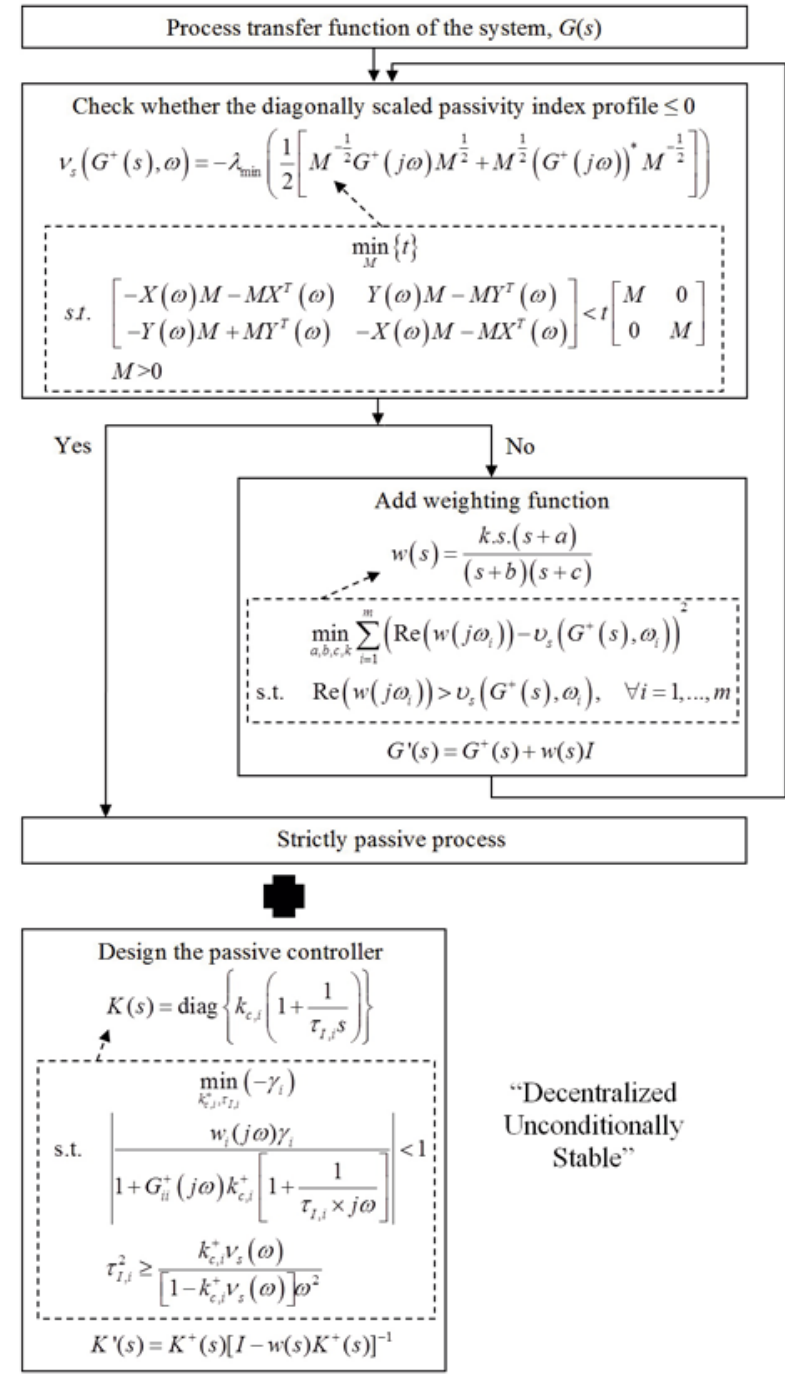

Figure 2. Generic concept of passivity controller

passivity index: using $v_{s}$ at a number of frequency points, $\omega . \lambda_{\min }$ as the minimum eigenvalue, and $G^{+}(s)$ represent the matrix product of process transfer function of both $G(s)$ and diagonal matrix $U$. The $U$ matrix is either 1 or -1 , which makes the diagonal signs of $G^{+}(s)$ positive. A diagonal and real matrix $M$ can be obtained by solving the optimization problem ${ }^{14}$. Variables $t, X$, and $Y$ are time, space of state variable, and space of output variable; respectively. When the resulting diagonal scaling passivity index profile is smaller than or equal to zero, it means the process is strictly passive. On the other hand, a weighting function of $w(s)$ is required to drive the process into a passive region through solving the optimization problem ${ }^{15}$. Also the parameters $a, b$, $c$, and $k$ should be determined correspondingly to the introduction of a weighting function; which can drive $G^{\prime}(s)$ to a strictly passive region.

Next, the passive controller $K^{\prime}(s)$, can be designed to achieve decentralized unconditional stability. The parameters $k_{c, i}^{+}$and $\tau_{I, i}$ are obtained through maximizing the sensitivity function of $\gamma_{i}$ with common optimization tools. The weighting function is also added to make the controller passive. $K^{+}(s)$ is the matrix product of $K(s)$ and the inverse of diagonal matrix $U$. The resulting stable closed-loop system was shown in Figure 3.

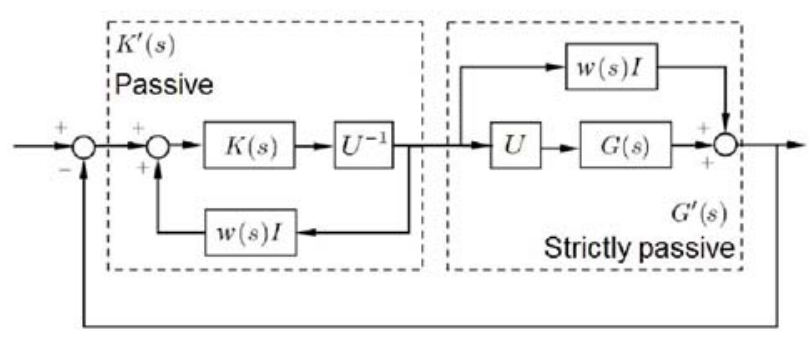

Figure 3. The resulting stable closed-loop system

\section{Passivity-based model predictive control}

The passivity-based MPC scheme is similar to the MPC method, as shown in Eq. (10). The following equation, which guarantees closed-loop stability, is added into the constraint ${ }^{13}$.

$u_{j}(k+i \mid k)^{T} y_{j}(k+i+1 \mid k) \leq-y_{j}(k+i+1 \mid k)^{T} y_{j}(k+i+1 \mid k)$

\section{RESULTS AND DISCUSSION}

The HEN example used in this work ${ }^{13}$ is shown in Figure 4.

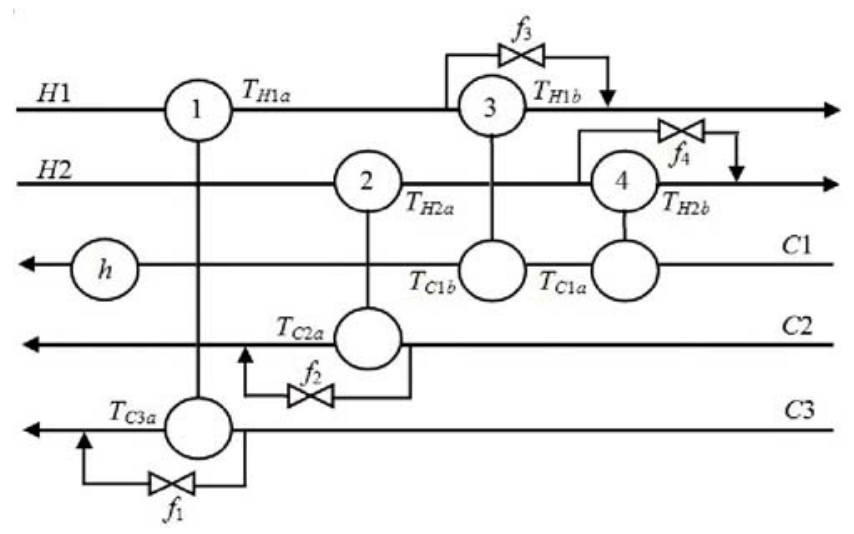

Figure 4. Control structure of heat exchanger network [16]

In this network, there are five target temperatures of $121.1,65.6,204.4,182.2$, and $204.4^{\circ} \mathrm{C}$, which are the outlet temperatures of streams $H 1, H 2, C 1, C 2$, and $C 3$, accordingly. The manipulations are bypass fractions $\left(f_{1}\right.$, $f_{2}, f_{3}$, and $\left.f_{4}\right)$ and heat duties of heater $\left(Q_{h}\right)$.

The simulations were carried out using Simulink, which is an extension of MATLAB programming language (R2011a). Disturbance rejection and set point tracking were tested to evaluate the stability and performance of the control system.

Five control loops in this HEN example are described as:

(1) $T_{H 1 \text { out }}-f_{3}$ loop: Outlet temperature of hot stream 1 is controlled by bypass fraction of heat exchanger No. 3 .

(2) $T_{H 2 \text { out }}-f_{4}$ loop: Outlet temperature of hot stream 2 is controlled by bypass fraction of heat exchanger No. 4 .

(3) $T_{C \text { lout }}-Q_{h}$ loop: Outlet temperature of cold stream 1 is controlled by heat duty of heater.

(4) $T_{C 2 \text { out }}-f_{2}$ loop: Outlet temperature of cold stream 2 is controlled by bypass fraction of heat exchanger No. 2 .

(5) $T_{\text {C3out }}-f_{1}$ loop: Outlet temperature of cold stream 3 is controlled by bypass fraction of heat exchanger No. 1 .

The process and the disturbance transfer function matrix of this HEN example is shown in Eqs. (13) and 
(14). The elements of the matrix are presented in the Appendix.

$G(s)=\left[\begin{array}{c}T_{H 1 \text { out }} \\ T_{H \text { 2out }} \\ T_{C \text { lout }} \\ T_{C 2 \text { out }} \\ T_{C 3 \text { out }}\end{array}\right]\left[\begin{array}{l}f_{1} \\ f_{2} \\ f_{3} \\ f_{4} \\ Q_{h}\end{array}\right]^{-1}=\left[\begin{array}{ccccc}G_{1,1} & G_{1,2} & G_{1,3} & G_{1,4} & 0 \\ 0 & G_{2,2} & 0 & G_{2,4} & 0 \\ 0 & G_{3,2} & 0 & 0 & 0 \\ G_{4,1} & 0 & 0 & 0 & 0 \\ G_{5,1} & G_{5,2} & G_{5,3} & G_{5,4} & G_{5,5}\end{array}\right]$

$G_{d}(s)=\left[\begin{array}{c}T_{H 1 \text { out }} \\ T_{H \text { out }} \\ T_{C \text { lout }} \\ T_{C 2 \text { out }} \\ T_{C 3 \text { out }}\end{array}\right]\left[\begin{array}{l}T_{H \text { lin }} \\ T_{H 2 \text { in }} \\ T_{C \text { lin }} \\ T_{C 2 \text { in }} \\ T_{C 3 \text { in }}\end{array}\right]^{-1}=\left[\begin{array}{ccccc}G_{d 1,1} & G_{d 1,2} & G_{d 1,3} & G_{d 1,4} & G_{d 1,5} \\ 0 & G_{d 2,2} & G_{d 2,3} & G_{d 2,4} & 0 \\ 0 & G_{d 3,2} & 0 & G_{d 3,4} & 0 \\ G_{d 4,1} & 0 & 0 & 0 & G_{d 4,5} \\ G_{d 5,1} & G_{d 5,2} & G_{d 5,3} & G_{d 5,4} & G_{d 5,5}\end{array}\right]$

\section{Proportional integral controller}

Tuning parameters of the PI controller were designed using an auto-tuning mode and are presented in Table 1.

Table 1. Proportional-integral tuning parameters

\begin{tabular}{|l|c|c|}
\hline Loop & $k_{c, i}$ & $\tau_{1, \mathrm{i}}[\mathrm{min}]$ \\
\hline$T_{\text {H1out }}-f_{3}$ & 142.8 & 3.41 \\
\hline$T_{\text {H2out }}-f_{4}$ & 127.8 & 3.45 \\
\hline$T_{\text {C1out }}-Q_{h}$ & 57.19 & 4.81 \\
\hline$T_{\text {C2out }}-f_{2}$ & 125.1 & 2.41 \\
\hline$T_{\text {C3out }}-f_{1}$ & 109.2 & 0.83 \\
\hline
\end{tabular}

\section{Model predictive controller}

Based on the transfer function model of Eqs. (13) and (14), model predictive controllers can be designed using a model predictive control toolbox in Simulink.

\section{Passivity controller}

Sequential to the passivity concept described in Figure 2 , the diagonal scaling passivity index was initially calculated. Figure 5 shows a resulting positive profile; meaning that this HEN example is a non-passive process and needs additional weighting function to spur it into the passive region.

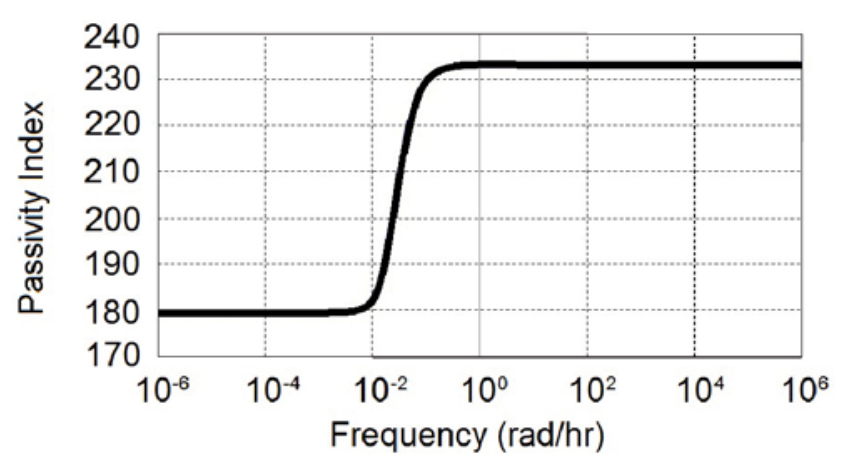

Figure 5. Diagonal scaling passivity index profile

The weighting function was calculated as the following:

$w(s)=\frac{1.1966 \times 10^{-10} s(s+0.0219)}{(s+0.0885)(s+0.0885)}$

Figure 6 shows the diagonal scaling passivity index after an additional weighting function was used. The profile results in a negative passive property.

To achieve closed-loop stability, the controller was designed via the optimization solution in Figure 2. The

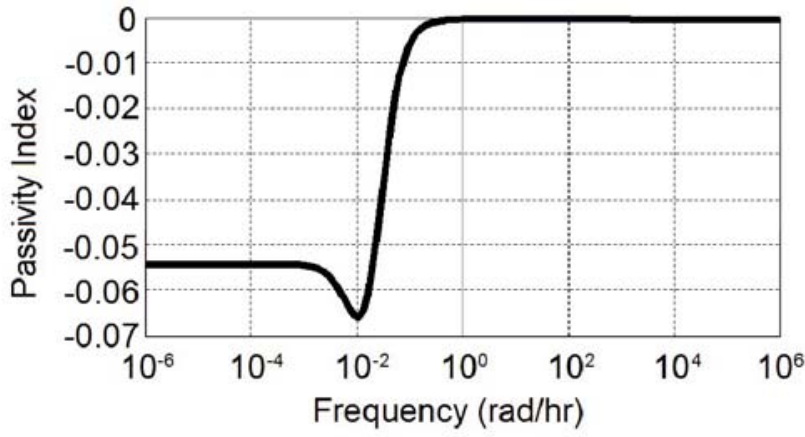

Figure 6. Diagonal scaling passivity index profile after adding weighting function

tuning parameters of each control-loop are shown in Table 2.

After supplemental weighting function was added to the controller to initiate a passive property, the parame-

Table 2. Passivity tuning parameters

\begin{tabular}{|l|c|c|}
\hline Loop & $k_{c, i}$ & $\tau_{1, \mathrm{i}}[\mathrm{min}]$ \\
\hline$T_{\text {H1 out }}-f_{3}$ & 75.58 & 6.63 \\
\hline$T_{\text {H2out }}-f_{4}$ & 106.2 & 4.76 \\
\hline$T_{C 1 \text { out }}-Q_{h}$ & 143.8 & 5.11 \\
\hline$T_{C 2 \text { out }}-f_{2}$ & 122.5 & 2.11 \\
\hline$T_{\text {C3out }}-f_{1}$ & 117.4 & 0.66 \\
\hline
\end{tabular}

ters presented in Eq. (16) were used to finally achieve control of the system.

\section{Passivity-based model predictive controller}

Based on the passive transfer function model of Eq. (17), passivity-based model predictive controllers could be designed using a MPC Design Task. Each element in the matrix is presented in the Appendix.

$$
\begin{aligned}
& K^{\prime}(s)_{T_{H 1 \text { out }}-f_{3}}=\frac{1.0329 s^{3}+2.2478 s^{2}+0.3738 s+0.01618}{4999 s^{3}+885 s^{2}+39 s} \\
& K^{\prime}(s)_{T_{H 2 \text { out }}-f_{4}}= \frac{4.796 s^{3}+17 s^{2}+2.8675 s+0.12524}{2999 s^{3}+531 s^{2}+24 s} \\
& K^{\prime}(s)_{T_{C 1 \text { out }}-Q_{h}}=\frac{0.15 s^{3}+1.527 s^{2}+0.267 s+0.012}{s^{3}+0.178 s^{2}+7.834 \times 10^{-8} s} \\
& K^{\prime}(s)_{T_{C 2 \text { out }}-f_{2}}=\frac{8.0662 s^{3}+18 s^{2}+2.919 s+0.1264}{4999 s^{3}+885 s^{2}+39 s} \\
& K^{\prime}(s)_{T_{C 3 \text { out }}-f_{1}}=\frac{3.23 s^{3}+17 s^{2}+2.8842 s+0.12652}{1999 s^{3}+359 s^{2}+16 s}
\end{aligned}
$$

$$
G^{\prime}(s)=G^{+}(s)+w(s) I=\left[\begin{array}{ccccc}
G_{1,1}^{\prime} & G_{1,2}^{\prime} & G_{1,3}^{\prime} & G_{1,4}^{\prime} & 0 \\
0 & G_{2,2}^{\prime} & 0 & G_{2,4}^{\prime} & 0 \\
0 & G_{3,2}^{\prime} & G_{3,3}^{\prime} & 0 & 0 \\
G_{4,1}^{\prime} & 0 & 0 & G_{4,4}^{\prime} & 0 \\
G_{5,1}^{\prime} & G_{5,2}^{\prime} & G_{5,3}^{\prime} & G_{5,4}^{\prime} & G_{5,5}^{\prime}
\end{array}\right]
$$




\section{Disturbance rejection test}

An inlet temperature of hot stream 2 was considered to be a disturbance in this case. Figure 7 reveals the dynamic responses to the change of a $+5 \%$ step disturbance occurring at the $5,000^{\text {th }}$ second and a $-5 \%$ step disturbance developing at the $10,000^{\text {th }}$ second.

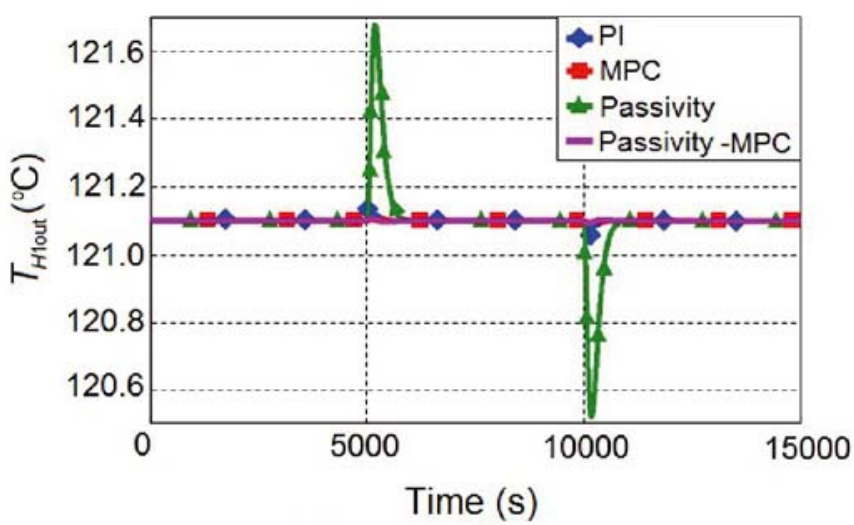

(a) Outlet temperature of hot stream 1

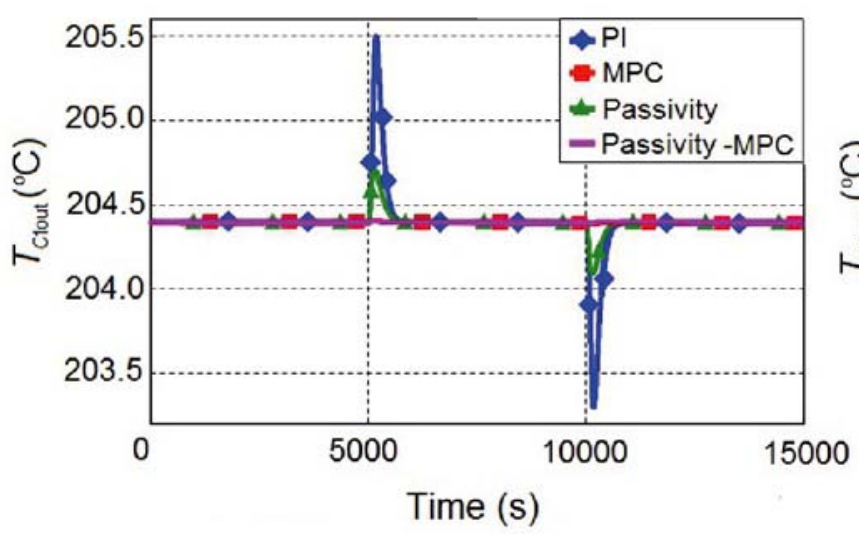

(c) Outlet temperature of cold stream 1
The results show that each controller can completely regulate output responses to reach their designated targets. The disturbance does not affect the outlet temperature of cold stream 3. For each controller, the settling time $\left(T_{s}\right)$ and percentage overshoot $(\mathrm{PO})$ of controlled variables $(\mathrm{CV})$ are concluded in Table 3.

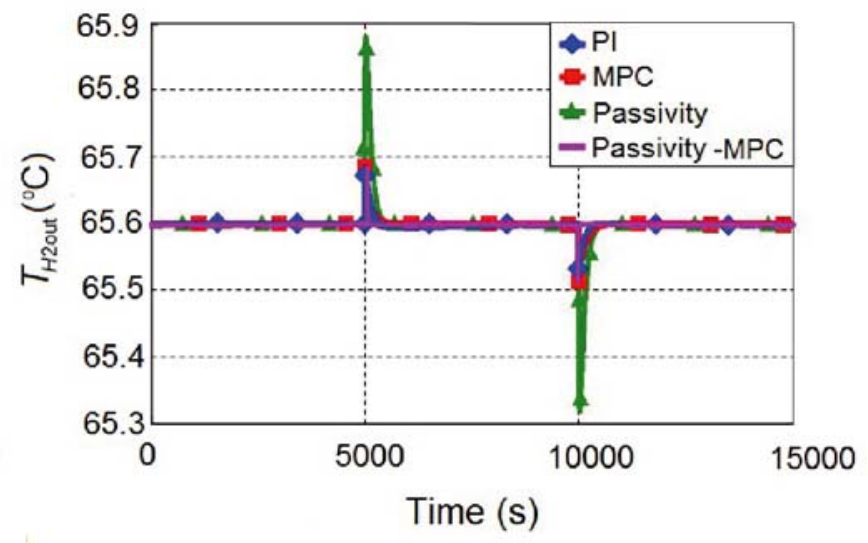

(b) Outlet temperature of hot stream 2

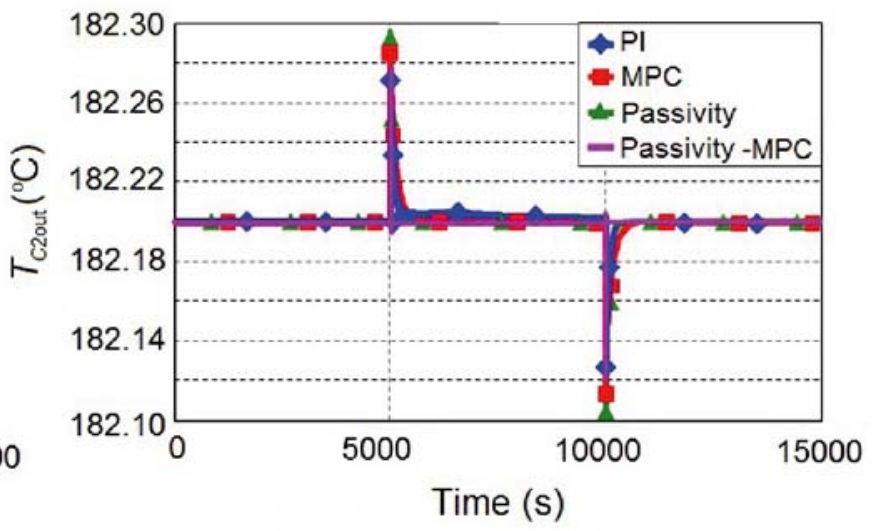

(d) Outlet temperature of cold stream ?

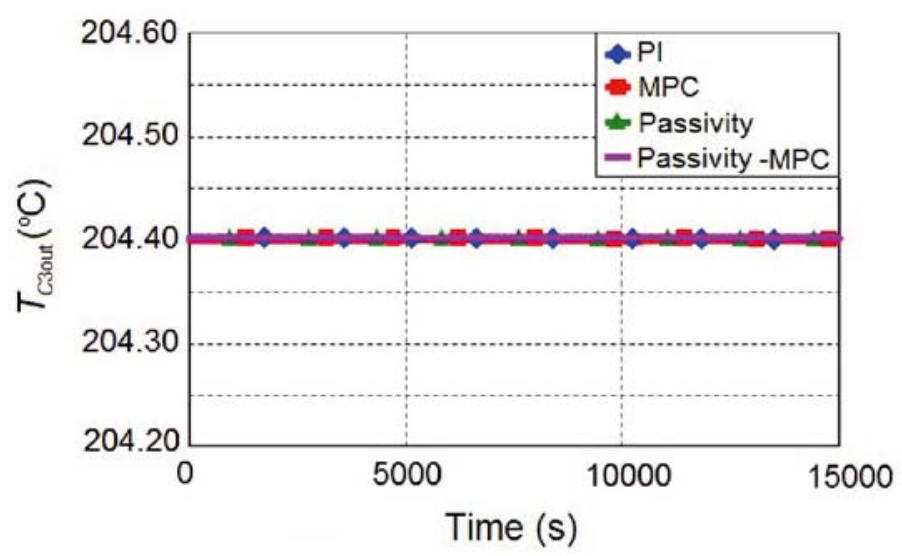

(e) Outlet temperature of cold stream 3

Figure 7. Performances of PI, MPC, Passivity, and Passivity-MPC controller to a $\pm 5 \%$ step change of inlet temperature of hot stream 2

Table 3. Performance measurement in case of disturbance rejection test

\begin{tabular}{|l|c|c|c|c|c|c|c|c|}
\hline \multirow{2}{*}{$\mathrm{CV}$} & \multicolumn{2}{|c|}{$\mathrm{PI}$} & \multicolumn{2}{c|}{ MPC } & \multicolumn{2}{c|}{ Passivity } & \multicolumn{2}{c|}{ Passivity-MPC } \\
\cline { 2 - 9 } & $T_{s}[\mathrm{~s}]$ & $\mathrm{PO}[\%]$ & $T_{s}[\mathrm{~s}]$ & $\mathrm{PO}[\%]$ & $T_{s}[\mathrm{~s}]$ & $\mathrm{PO}[\%]$ & $T_{s}[\mathrm{~s}]$ & $\mathrm{PO}[\%]$ \\
\hline$T_{\text {H1out }}$ & 512 & 0.0190 & 512 & 0.0038 & 994 & 0.4785 & 241 & 0.0069 \\
\hline$T_{\text {H2out }}$ & 518 & 0.1212 & 1054 & 0.1377 & 714 & 0.4316 & 75 & 0.1286 \\
\hline$T_{\text {C1 out }}$ & 721 & 0.5443 & 315 & 0.0044 & 766 & 0.1510 & 240 & 0.0027 \\
\hline$T_{\text {C2out }}$ & 362 & 0.0421 & 785 & 0.0488 & 317 & 0.0535 & 41 & 0.0425 \\
\hline$T_{\text {C30ut }}$ & - & - & - & - & - & - & - & - \\
\hline
\end{tabular}


Settling time is the time required for the response to reach and stay within a specified tolerance band $( \pm 5 \%)$ of its final value. Percentage of Overshoot refers to the normalized difference between the response peak value and the final value.

The settling time is measures of the speed of the response, whereas the percentage of overshoot is related to the quality of the response. It is evident that the passivity-based MPC's performance is the best method employed in this case, due to its rapid settling time and the smallest percentage of overshoot. The controlled response of the PI and the passivity-based MPC procedures are actually comparable, but PI's settling time is considerably larger. Whereas, the MPC and passivity controller's performances are satisfactory, based on the acceptable values of settling time and percentage overshoot.

\section{Setpoint tracking test}

An outlet temperature of hot stream 2's setpoint is considered altered. Figure 8 shows the dynamic responses to the change of a $+5 \%$ step setpoint beginning at the 5,000 th second and a $-5 \%$ step setpoint occurring at the 10,000th second.

The experimental results indicate that each controller can fully control output responses to reach their intended targets. The setpoint changing does not affect the outlet temperature of cold streams 2 and 3 . For each controller, the values of settling time $\left(T_{s}\right)$ and percentage overshoot $(\mathrm{PO})$ of controlled variables $(\mathrm{CV})$ are presented in Table 4.

Resulting observations indicate that the MPC model provides the best performance because its settling time and PO are better than those of other controllers. The performances of the PI, passivity, and passivity-based

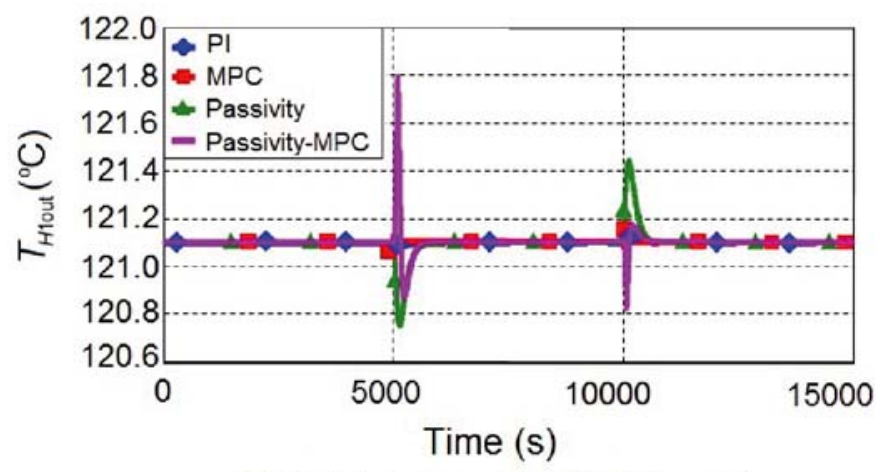

(a) Outlet temperature of hot stream 1

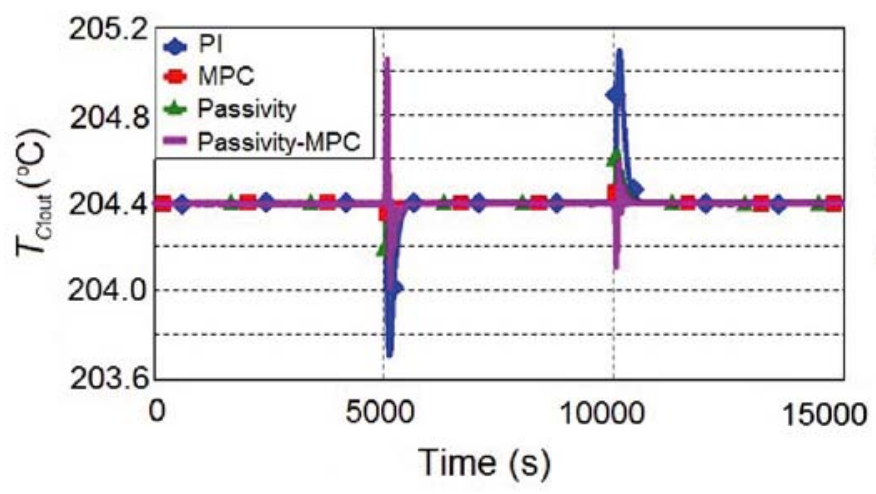

(c) Outlet temperature of cold stream 1

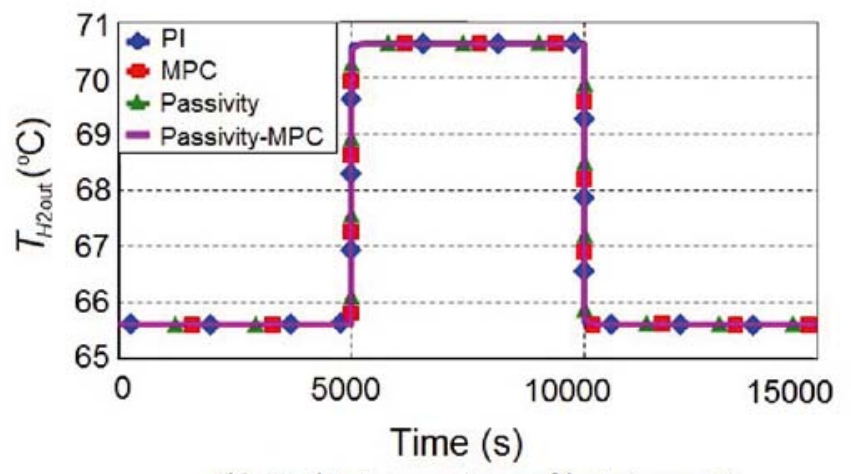

(b) Outlet temperature of hot stream 2

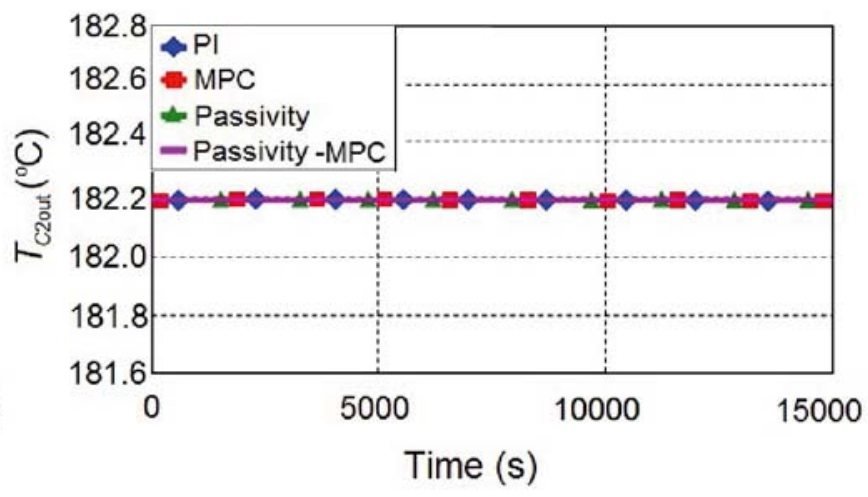

(d) Outlet temperature of cold stream 2

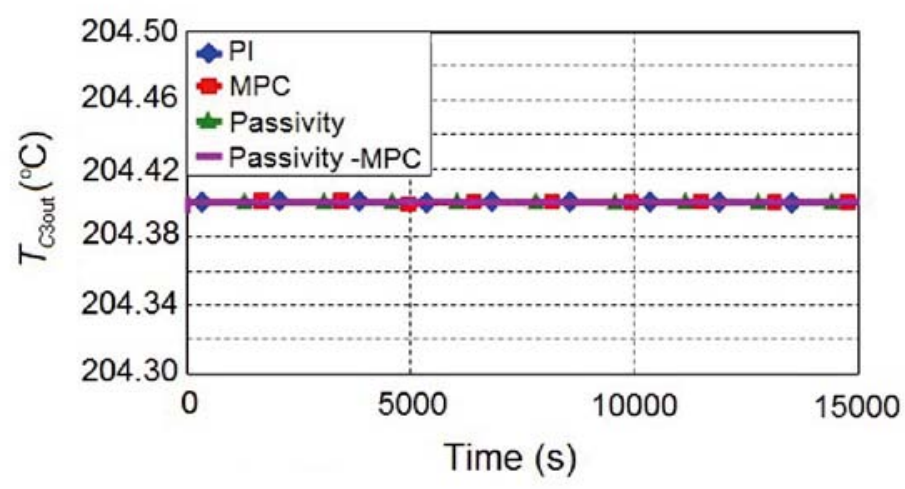

(e) Outlet temperature of cold stream 3

Figure 8. Performance of PI, MPC, Passivity, and Passivity-MPC controllers to set point tracking of outlet temperature of hot stream 2 
Table 4. Performance measurement in case of set point tracking test

\begin{tabular}{|l|c|c|c|c|c|c|c|c|}
\hline \multirow{2}{*}{$\mathrm{CV}$} & \multicolumn{2}{|c|}{$\mathrm{PI}$} & \multicolumn{2}{c|}{$\mathrm{MPC}$} & \multicolumn{2}{c|}{ Passivity } & \multicolumn{2}{c|}{ Passivity-MPC } \\
\cline { 2 - 9 } & $T_{s}[\mathrm{~s}]$ & $\mathrm{PO}[\%]$ & $T_{s}[\mathrm{~s}]$ & $\mathrm{PO}[\%]$ & $T_{s}[\mathrm{~s}]$ & $\mathrm{PO}[\%]$ & $T_{s}[\mathrm{~s}]$ & $\mathrm{PO}[\%]$ \\
\hline$T_{\text {H1out }}$ & 331 & 0.0148 & 210 & 0.0265 & 753 & 0.2536 & 723 & 0.5756 \\
\hline$T_{\text {H2out }}$ & - & - & - & - & - & - & - & - \\
\hline$T_{\text {C10ut }}$ & 633 & 0.3434 & 241 & 0.0155 & 663 & 0.1165 & 587 & 0.3232 \\
\hline$T_{\text {C2out }}$ & - & - & - & - & - & - & - & - \\
\hline$T_{\text {C30ut }}$ & - & - & - & - & - & - & - & - \\
\hline
\end{tabular}

MPC controllers are also sufficient, due to the acceptable values of their settling time and percentage overshoot.

\section{CONCLUSIONS}

The performances of several advanced control techniques were examined and compared for a case of heat exchanger network. The passivity-based MPC controller is the best to reject the disturbance. The MPC system was also shown to be the best controller to track the setpoint. The values of an average settling time of proportional integral, model predictive, passivity, and passivity-based model predictive controllers were observed as 513, 529, 687, and 324 seconds, respectively. The average percentage overshoot values for proportional integral, model predictive control, passivity, and passivity-based model predictive controllers were found as $0.1473 \%, 0.0429 \%$, $0.1960 \%$, and $0.1187 \%$, also respectively. Therefore, it was proved that the passivity-based model predictive controller is the best system choice in this research.

\section{ACKNOWLEDGEMENTS}

This work was supported by the Royal Golden Jubilee Ph.D. Program (Grant No. PHD/0058/2549).

\section{APPENDIX}

The elements of process transfer function matrix

$$
\begin{aligned}
G_{1,1} & =\frac{73.88 s^{2}+2542 s+84.68}{s^{4}+77.29 s^{3}+1477 s^{2}+75.17 s+0.9516}, \\
G_{1,2} & =\frac{10.36}{s^{6}+116.4 s^{5}+4392 s^{4}+5.418 \times 10^{4} s^{3}+3695 s^{2}+84.14 s+0.6384}, \\
G_{1,3} & =\frac{81.4 s^{2}+2797 s+37.92}{s^{2}+34.39 s+0.806}, \\
G_{1,4} & =\frac{-5.699}{s^{4}+64.86 s^{3}+1049 s^{2}+47.06 s+0.5272}, \\
G_{2,2} & =\frac{92.33 s^{2}+2815 s+82.67}{s^{4}+81.98 s^{3}+1572 s^{2}+70.6 s+0.7921}, \\
G_{2,4} & =\frac{50.8 s^{2}+1547 s+20.97}{s^{2}+30.48 s+0.6541}, \\
G_{3,2} & =\frac{-233.2 s^{2}-1.201 \times 10^{4} s-189.9}{s^{2}+51.51 s+1.211}, \\
G_{4,1} & =\frac{-222.3 s^{2}-9532 s-188.4}{s^{2}+42.9 s+1.181}, \\
G_{5,1} & =\frac{-1.362}{s^{5}+77.31 s^{4}+1479 s^{3}+115.2 s^{2}+2.991 s+0.02582},
\end{aligned}
$$$$
G_{5,2}=\frac{0.03274 s+0.5627}{s^{7}+116.4 s^{6}+4.395 s^{5}+5.43 \times 10^{4} s^{4}+5165 s^{3}+184.4 s^{2}+2.921 s+0.01732},
$$

$$
\begin{aligned}
G_{5,3} & =\frac{-1.5}{s^{3}+34.41 s^{2}+1.739 s+0.02187}, \\
G_{5,4} & =\frac{-0.01801 s-0.3096}{s^{5}+64.89 s^{4}+1051 s^{3}+75.53 s^{2}+1.804 s+0.0143}, \\
G_{5,5} & =\frac{0.002381}{s+0.02713},
\end{aligned}
$$

The elements of disturbance transfer function matrix $G_{d 1,1}=\frac{0.01978 s^{3}+1.105 s^{2}+14.61 s+0.4861}{s^{4}+77.29 s^{3}+1477 s^{2}+75.17 s+0.9516}$,

$G_{d 1,2}=\frac{0.001776 s+0.04571}{s^{6}+116.4 s^{5}+4392 s^{4}+5.418 \times 10^{4} s^{3}+3695 s^{2}+84.14 s+0.6384}$,

$G_{d 1,3}=\frac{0.006319 s+0.09626}{s^{4}+64.86 s^{3}+1049 s^{2}+47.06 s+0.5272}$,

$G_{d 1,4}=\frac{0.02221}{s^{6}+116.4 s^{5}+4392 s^{4}+5.418 \times 10^{4} s^{3}+3695 s^{2}+84.14 s+0.6384}$,

$G_{d 1,5}=\frac{0.1662 s^{2}+5.717 s+0.1905}{s^{4}+77.29 s^{3}+1477 s^{2}+75.17 s+0.9516}$,

$G_{d 2,2}=\frac{0.01583 s^{3}+0.8901 s^{2}+12.44 s+0.3648}{s^{4}+81.98 s^{3}+1572 s^{2}+70.6 s+0.7921}$,

$G_{d 2,3}=\frac{0.2064}{s^{2}+30.48 s+0.6541}$,

$G_{d 2,4}=\frac{0.198 s^{2}+6.036 s+0.1773}{s^{4}+81.98 s^{3}+1572 s^{2}+70.6 s+0.7921}$,

$G_{d 3,2}=\frac{0.4072}{s^{2}+51.51 s+1.211}$,

$G_{d 3,4}=\frac{0.007694 s+0.1982}{s^{2}+51.51 s+1.211}$,

$G_{d 4,1}=\frac{0.4238}{s^{2}+42.9 s+1.181}$,

$G_{d 4,5}=\frac{0.007756 s+0.1665}{s^{2}+42.9 s+1.181}$,

$G_{d 5,1}=\frac{0.0003646 s+0.007817}{s^{5}+77.31 s^{4}+1479 s^{3}+115.2 s^{2}+2.991 s+0.02582}$,

$G_{d 5,2}=\frac{5.612 \times 10^{-6} s^{2}+0.0002409 s+0.002483}{s^{7}+116.4 s^{6}+4395 s^{5}+5.43 \times 10^{4} s^{4}+5165 s^{3}+184.4 s^{2}+2.921 s+0.01732}$,

$G_{d 5,3}=\frac{1.997 \times 10^{-5} s^{2}+0.0006475 s+0.005229}{s^{5}+64.89 s^{4}+1051 s^{3}+75.53 s^{2}+1.804 s+0.0143}$,

$G_{d 5,4}=\frac{7.019 \times 10^{-5} s+0.001207}{s^{7}+116.4 s^{6}+4395 s^{5}+5.43 \times 10^{4} s^{4}+5165 s^{3}+184.4 s^{2}+2.921 s+0.01732}$,

$G_{d 5,5}=\frac{0.003063}{s^{5}+77.31 s^{4}+1479 s^{3}+115.2 s^{2}+2.991 s+0.02582}$, 
The elements of passive transfer function matrix $G_{1,1}^{\prime}=\frac{1.197 \times 10^{-10} s^{6}+9.251 \times 10^{-9} s^{5}+73.88 s^{4}+2555 s^{3}+535.2 s^{2}+34.82 s+0.6605}{s^{6}+77.47 s^{5}+1491 s^{4}+337.2 s^{3}+25.78 s^{2}+0.7548 s+0.007422}$,

$$
\begin{aligned}
& G_{1,2}^{\prime}=\frac{10.36}{s^{6}+116.4 s^{5}+4392 s^{4}+54180 s^{3}+3695 s^{2}+84.14 s+0.6384}, \\
& G_{1,3}^{\prime}=\frac{81.4 s^{2}+2797 s+37.92}{s^{2}+34.39 s+0.806} \\
& G_{1,4}^{\prime}=\frac{-5.699}{s^{4}+64.86 s^{3}+1049 s^{2}+47.06 s+0.5272}, \\
& G_{2,2}^{\prime}=\frac{1.197 \times 10^{-10} s^{6}+9.812 \times 10^{-9} s^{5}+92.33 s^{4}+2831 s^{3}+581.6 s^{2}+36.59 s+0.6448}{s^{6}+82.16 s^{5}+1587 s^{4}+349.5 s^{3}+25.55 s^{2}+0.6909 s+0.006178}, \\
& G_{2,4}^{\prime}=\frac{50.8 s^{2}+1547 s+20.97}{s^{2}+30.48 s+0.6541} \\
& G_{3,2}^{\prime}=\frac{-233.2 s^{2}-1.201 \times 10^{4} s-189.9}{s^{2}+51.51 s+1.211}, \\
& G_{3,3}^{\prime}=\frac{1.197 \times 10^{-10} s^{2}+2.62 \times 10^{-12} s}{s^{2}+0.177 s+0.0078}, \\
& G_{4,1}^{\prime}=\frac{-222.3 s^{2}-9532 s-188.4}{s^{2}+42.9 s+1.181} \\
& G_{5,1}^{\prime}=\frac{-1.362}{s^{5}+77.31 s^{4}+1479 s^{3}+115.2 s^{2}+2.991 s+0.02582}, \\
& G_{5,2}^{\prime}=\frac{0.03274 s+0.5627}{s^{7}+116.4 s^{6}+4.395 s^{5}+5.43 \times 10^{4} s^{4}+5165 s^{3}+184.4 s^{2}+2.921 s+0.01732}, \\
& G_{5,3}^{\prime}=\frac{-1.5}{s^{3}+34.41 s^{2}+1.739 s+0.02187}, \\
& G_{5,4}^{\prime}=\frac{-0.01801 s-0.3096}{s^{5}+64.89 s^{4}+1051 s^{3}+75.53 s^{2}+1.804 s+0.0143}, \\
& G_{5,5}^{\prime}=\frac{1.197 \times 10^{-10} s^{3}+0.002381 s^{2}+0.0004214 s+1.857 \times 10^{-5}}{s^{3}+0.2041 s^{2}+0.0126 s+0.0002116},
\end{aligned}
$$

\section{LITERATURE CITED}

1. Westphalen, D.L., Young, B.R. \& Svrcek, W.Y. (2003). A Controllability Index for Heat Exchanger Networks. Ind. Eng. Chem. Res. 42(20), 4659-4667. DOI: 10.1021/ie020893z.

2. Seborg, D.E., Edgar, T.F., Mellichamp, D.A. \& Doyle III, F.J. (2011). Process dynamics and control (3rd ed.). New Jersey, USA: John Wiley \& Sons, Inc.

3. Grüne, L. \& Pannek, J. (2011). Nonlinear Model Predictive Control: Theory and Algorithms. London United Kingdom: Springer-Verlag London Limited.

4. Bakosova, M. \& Oravec, J. (2014). Robust model predictive control for heat exchanger network. Appl. Therm. Eng. 73, 924-930. DOI: 10.1016/j.applthermaleng. 2014.08.023.

5. Rene, A.S. (2016). Model Predictive Control of District Heating Systems. Master dissertation, Norwegian University of Science and Technology, Trondheim, Norway.

6. Heidarinejad, M., Liu, J. \& Christofides, P.D. (2011). Lyapunov-based economic model predictive control of nonlinear systems. In American Control Conference, 29 June - 1 July 2011 (pp. 5195-5200). San Francisco, CA, USA.

7. Liu, J., Munoz de la Pena, D., Christofides, P.D. \& Davis, J.F. (2009). Lyapunov-based model predictive control of nonlinear systems subject to time-varying measurement delays. Int. J. Adapt. Control Signal Process. 23(8), 788-807. DOI: 10.1002/acs.1085.

8. Pukdeboon, C. (2011). A Review of Fundamentals of Lyapunov Theory. J. Appl. Sci. 10(2), 55-61.

9. Bao, J., Wan, F.Y., Lee, P.L. \& Zhou, W.B. (1996). New frequency-domain phase-related properties of MIMO LTI passive systems and robust controller synthesis. In $13^{\text {th }}$ IFAC
World Congress, 30 June - 5 July 1996 (pp. 405-410). San Francisco, CA, USA.

10. Bao, J., Lee, P.L., Wan, F.Y. \& Zhou, W.B. (2000). A New Approach to Decentralized Process Control Using Passivity and Sector Stability Conditions. Chem. Eng. Commun. 182(1), 213-237. DOI: 10.1080/00986440008912835.

11. Bao, J., Zhang, W.Z. \& Lee, P.L. (2002). Passivity-Based Decentralized Failure-Tolerant Control. Ind. Eng. Chem. Res. 41(23), 5702-5715. DOI: 10.1021/ie0201314.

12. Zhang, W.Z., Bao, J. \& Lee, P.L. (2002). Decentralized Unconditional Stability Conditions Based on the Passivity Theorem for Multi-loop Control Systems. Ind. Eng. Chem. Res. 41(6), 1569-1578. DOI: 10.1021/ie001037v.

13. Raff, T., Ebenbauer, C. \& Allgower, P. (2007). Nonlinear Model Predictive Control: A Passivity-based Approach. In I.R. Findeisen, F. Allgower \& L.T. Biegler (Eds.), Assessment and Future Directions of Nonlinear Model Predictive Control (pp. 151-162). Germany: Springer Berlin Heidelberg.

14. Bao, J., Zhang, W.Z. \& Lee, P.L. (2000). A Passivity-based Approach to Multi-loop PI Controller Tuning. In $6^{\text {th }}$ International Conference on Control, Automation, Robotics and Vision, 5-8 December 2000 (paper 178). Singapore.

15. Bao, J. \& Lee, P.L. (2007). Process Control: The Passive Systems Approach. United Kingdom: Springer-Verlag London Limited.

16. Pariyani, A., Gupta, A. \& Ghosh, P. (2006). Design of heat exchanger networks using randomized algorithm. Comput. Chem. Eng. 30(6-7), 1046-1053. DOI: 10.1016/j.compchemeng.2006.01.005. 